\title{
Exposure to human alveolar lining fluid enhances Mycobacterium bovis BCG vaccine efficacy against Mycobacterium tuberculosis infection in a $\mathrm{CD}^{+}{ }^{+}$T-cell-dependent manner
}

\author{
JI Moliva ${ }^{1,2}$, AP Hossfeld ${ }^{1}$, CH Canan ${ }^{1}$, V Dwivedi ${ }^{1}$, MD Wewers ${ }^{3}$, G Beamer $^{4}$, J Turner ${ }^{1}$ and JB Torrelles $^{1}$
}

Current tuberculosis (TB) treatments include chemotherapy and preventative vaccination with Mycobacterium bovis Bacillus Calmette-Guérin (BCG). In humans, however, BCG vaccination fails to fully protect against pulmonary TB. Few studies have considered the impact of the human lung mucosa (alveolar lining fluid (ALF)), which modifies the Mycobacterium tuberculosis (M.tb) cell wall, revealing alternate antigenic epitopes on the bacterium surface that alter its pathogenicity. We hypothesized that ALF-induced modification of BCG would induce better protection against aerosol infection with M.tb. Here we vaccinated mice with ALF-exposed BCG, mimicking the mycobacterial cell surface properties that would be present in the lung during M.tb infection. ALF-exposed BCG-vaccinated mice were more effective at reducing $M$.tb bacterial burden in the lung and spleen, and had reduced lung inflammation at late stages of M.tb infection. Improved BCG efficacy was associated with increased numbers of memory CD8 ${ }^{+} \mathrm{T}_{\text {cells, and } \mathrm{CD}}{ }^{+} \mathrm{T}$ cells with the potential to produce interferon- $\gamma$ in the lung in response to $M$.tb challenge. Depletion studies confirmed an essential role for $\mathrm{CD}^{+}$T cells in controlling M.tb bacterial burden. We conclude that ALF modifications to the M.tb cell wall in vivo are relevant in the context of vaccine design.

\section{INTRODUCTION}

Mycobacterium tuberculosis (M.tb) causes significant morbidity and mortality around the world every year. Tuberculosis (TB) is now the leading cause of death attributed to a single infectious organism, surpassing the human immunodeficiency virus. ${ }^{1}$ Mathematical models predict $\sim 1.7$ billion individuals, a quarter of the world's population, to be infected with $M$.tb in a latent state serving as a large reservoir for the disease. ${ }^{2}$ Current chemotherapy against TB, although effective, has led to the rise of drug-resistant strains, making it more difficult to curtail this disease. ${ }^{1}$ Thus, the best approach to contain, and potentially eradicate, TB may lie in the development of an effective vaccine. Mycobacterium bovis Bacille de Calmette et Guérin (BCG) is the only vaccine currently supported by the World Health
Organization for the prevention of TB. However, the efficacy of BCG at preventing pulmonary TB is highly variable, ${ }^{3,4}$ and its protective immunity in humans only appears to last for 10-15 years. ${ }^{5}$ Despite many efforts to develop new effective TB vaccines over the past few decades, these approaches have resulted in little success. $3,4,6$

During the natural course of infection with M.tb, bacilli are inhaled and deposited in the alveolar sacs of the lung ${ }^{7}$ where they are bathed in alveolar lining fluid (ALF). ${ }^{8} \mathrm{ALF}$ is composed of surfactant lipids and its associated proteins, as well as of an aqueous hypophase rich in innate host defense molecules such hydrolytic enzymes, ${ }^{9}$ complement proteins, ${ }^{10}$ surfactant proteins $\mathrm{A}$ and $\mathrm{D},{ }^{11}$ and immunoglobulins. ${ }^{12}$ We have shown that interaction with ALF attenuates M.tb pathogenicity

${ }^{1}$ Department Microbial Infection and Immunity, College of Medicine (COM), The Ohio State University (OSU), Columbus, Ohio, USA. ${ }^{2}$ Biomedical Sciences Graduate Program, COM, OSU, Columbus, Ohio, USA. ${ }^{3}$ Department Internal Medicine, Pulmonary, Critical Care and Sleep Medicine Division, COM, OSU, Columbus, Ohio, USA and ${ }^{4}$ Department Infectious Diseases and Global Health, Cummings School of Veterinary Medicine, Tufts University, North Grafton, Massachussetts, USA. Correspondence: J Turner (joanneturner@txbiomed.org) or JB Torrelles (jtorrelles@txbiomed.org)

Both corresponding authors contributed equally to this study.

Received 7 April 2017; accepted 7 August 2017; published online 20 September 2017. doi:10.1038/mi.2017.80 
in vitro, ${ }^{9,13,14}$ likely due to the action of hydrolytic enzymes removing cell wall peripheral lipids such as mannose-capped lipoarabinomannan and trehalose dimycolate. ${ }^{9}$ Thus, exposure to human ALF modifies M.tb, reducing virulence factors and exposing new antigenic motifs on its surface before encountering and infecting host cells. Such changes modify uptake and phagocytosis by host cells, ${ }^{9,13,14}$ which initiate innate immune responses to M.tb that we consider to be influential in the generation of appropriate adaptive immune responses in vivo. It is therefore plausible to hypothesize that immunogenic cell wall motifs and virulence factors associated with M.tb are affected by M.tb's contact with ALF during infection.

In this study, we determined whether the underlying mechanism behind poor BCG efficacy may be due to discrepancies associated with the natural route of infection with $M . t b$ via the lung, vs. inoculation with BCG via the skin. We hypothesized that ALF-exposed BCG would generate an immune response against similar motifs that are accessible to the immune system during $M . t b$ infection in the lung, resulting in improved control of M.tb during challenge. We identified differences in immune responses to ALF-exposed BCG vaccination in the lung, particularly within the $\mathrm{CD} 8^{+} \mathrm{T}$-cell subset. When challenged with M.tb, ALF-exposed BCG vaccination led to a significant decrease in M.tb bacterial burden, reduced pulmonary inflammation, and extended survival in C57BL/6J mice. The reduction in bacterial burden was dependent on $\mathrm{CD}^{+}$T-cell responses and was associated with increased interferon- $\gamma$ (IFN $\gamma$ ) in the lung. Hence, we provide proof of principle that changes on the BCG cell wall surface, akin to the ones observed by M.tb after exposure to human ALF, have the potential to generate superior host immune responses and induce better protection against infection. Our studies highlight the importance of considering the properties of human ALF when developing an effective vaccine against $\mathrm{TB}$.

\section{RESULTS}

Vaccination with ALF-exposed BCG reduces bacterial burden in the lung and spleen and extends survival of mice C57BL/6J mice were vaccinated with either vehicle (mockvaccinated; no BCG), NaCl-exposed BCG, or ALF-exposed BCG. Six weeks after vaccination, all groups were infected with a low-dose aerosol of ALF-exposed M.tb (the same ALF used for BCG vaccination). The outcome of infection with ALF-exposed $M . t b$, in terms of lung bacterial burden, was similar to that of conventional laboratory M.tb strains (Supplementary Figure S1 online). ALF-exposed, and not broth grown M.tb, was specifically used to model the composition of the M.tb cell wall after exposure to human ALF. We observed similar biochemical changes to the BCG cell wall as we observed previously with ALF-exposed M.tb, with significant decreases in the amount of trehalose-6,6-dimycolate (TDM) and mannose-capped lipoarabinomannan (ManLAM) remaining on the BCG cell wall (Supplementary Figure S2A,B). ${ }^{9}$ Mice were killed at 14 days post infection (DPI) to specifically address whether ALFexposed BCG vaccination accelerated the generation of adaptive immunity relative to that observed with BCG
(Figure 1a). As expected, $\mathrm{NaCl}$-exposed BCG vaccination (gray bars) led to an expected moderate, yet significant, reduction in $M . t b$ colony-forming unit (CFU) in the lung after only 14 DPI (Figure 1b), indicating some acceleration of adaptive immunity in BCG-vaccinated mice relative to nonvaccinated controls. We did not anticipate a major reduction in M.tb CFU in the lung and spleen of BCG-vaccinated mice at 14 DPI, as the typical $1.0-\log _{10}$ reduction is not observed until $21-$ 30 DPI. ${ }^{15}$ In contrast to mice receiving $\mathrm{NaCl}$-exposed BCG, vaccination with ALF-exposed BCG (black bars) resulted in a superior early reduction in M.tb CFU in the lung and a 0.56 $\log _{10}$ reduction beyond that afforded by NaCl-exposed BCG. ALF-exposed BCG also conferred an additional $0.8-\log _{10}$ protection against dissemination, as indicated by a significant decrease in bacterial burden in the spleen (Figure 1b), although $\mathrm{CFU}$ were close to the level of accurate detection in this organ. Our data demonstrate that modification of BCG through exposure to ALF can further boost the protective efficacy of BCG in vivo, allowing for more rapid control of $M$.tb in the lung as demonstrated by significantly less M.tb CFUs at 14 DPI.

We also determined M.tb CFU at 250 DPI to assess the ability of ALF-exposed BCG to provide long-term control of M.tb infection and its impact on development of progressive disease. ${ }^{16} \mathrm{NaCl}$-exposed BCG was ineffective at conferring a reduction of M.tb CFU in the lung or spleen at 250 DPI (Figure 1c), similar to several studies that have shown limited long-term protection by BCG. ${ }^{17}$ In contrast, ALF-exposed BCG vaccination resulted in a significant reduction in $M$.tb CFU at 250 DPI (0.56- $\log _{10}$ protection) compared with vehicle control mice (Figure 1c). This trend was also statistically significant in the spleen (Figure 1c). Vaccination with ALF-exposed BCG also significantly extended survival (Figure 1d) to an average of 71.0 weeks, with some mice surviving $\sim 80.0$ weeks, demonstrating superior ability of ALF-exposed BCG to reduce TB severity. The median survival of vehicle-treated mice was 51.0 weeks, which is in line with previously published data $^{16}$ and $\mathrm{NaCl}$-exposed $\mathrm{BCG}$ vaccinated mice had a median survival of 64.50 weeks. Overall, exposure of BCG to ALF results in a vaccine that can generate accelerated M.tb control (Figure 1b; early reduction in $\mathrm{CFU}$ ) combined with the capacity to sustain control of M.tb for an extended period of time (Figure 1c; late reduction in CFUs), which ultimately extends survival by 20.0 weeks relative to non-vaccinated mice (and an additional 6.0 weeks beyond $\mathrm{NaCl}$-exposed BCG) (Figure 1d).

\section{Vaccination with ALF-exposed BCG reduces pulmonary inflammation}

To determine the capacity of ALF-exposed BCG to reduce severity of infection and progression of disease, we assessed the degree of tissue involvement through quantification of cellular aggregation relative to the total size of the lung. At 14 DPI (Figure 2a) we observed no statistically significant difference in tissue involvement between the three groups studied. However, both NaCl- and ALF-exposed BCG-vaccinated mice had moderately more cellular infiltration (Figure 2b), 
a

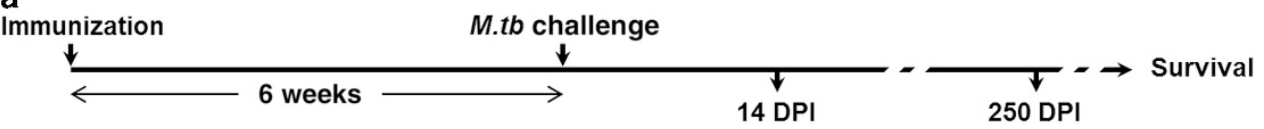

b

DPI 14

C

$250 \mathrm{DPI}$

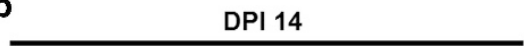

DPI 250
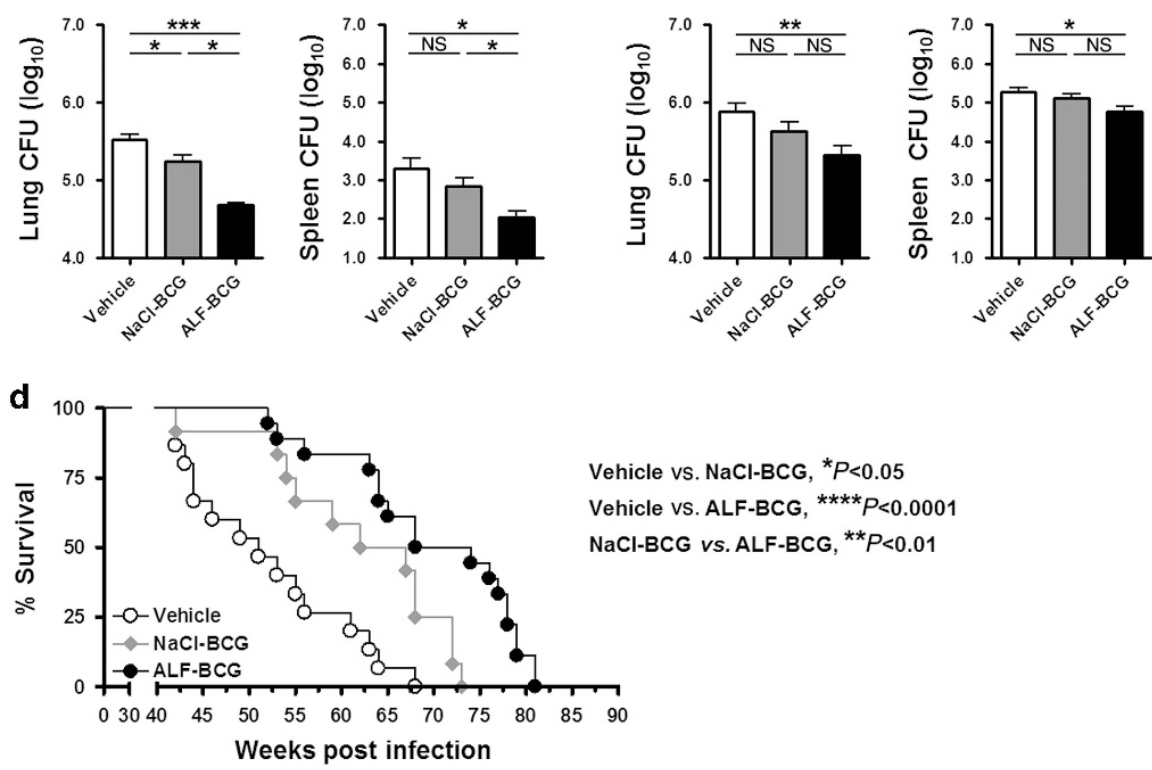

Figure 1 Vaccination with alveolar lining fluid (ALF)-exposed Bacillus Calmette-Guérin (BCG) reduces Mycobacterium tuberculosis (M.tb) bacterial burden in the lung and spleen of C57BL/6J mice and extends survival. (a) Experimental design. (b and c) C57BL/6J mice were vaccinated with NaClexposed BCG (NaCl-BCG; gray bars) or ALF-exposed BCG (ALF-BCG; black bars), or left unvaccinated (vehicle control; open bars). Six weeks later, mice were infected with a low-dose aerosol of $M$.tb. C57BL/6J mice were killed at 14 and 250 days post infection (DPI) and M.tb colony-forming unit (CFU) determined in the lung and spleen. Representative experiment from $n=3$ with 4-5 mice per group per time-point studied, mean \pm s.e.m.; one-way analysis of variance (ANOVA) with Tukey's post hoc test, ${ }^{\star} P<0.05$, ${ }^{* *} P<0.01$, and ${ }^{* * *} P<0.001$; NS: not significant. (d) C57BL/6J mice were vaccinated with vehicle (open circles), NaCl-exposed BCG (gray diamonds), or ALF-exposed BCG (black circles) and challenged with M.tb 6 weeks later. Survival was monitored across a period of 85 weeks. Mice were killed when they met the exclusion criteria documented in animal care and use protocols. Mice in the vehicle group $(n=15)$ displayed a mean survival of 50.00 weeks. NaCl-BCG $(n=12)$-vaccinated mice had a mean survival of 64.50 weeks. ALF-BCG ( $n=18$ )-vaccinated mice had a mean survival of 71.00 weeks. Pooled data from $n=3$ with $4-6$ mice per group (12-18 total mice per group), mean \pm s.e.m.; log-rank test, ${ }^{*}$ vehicle vs. NaCl-BCG $(P=0.0111),{ }^{* * *}$ vehicle vs. ALF-BCG $(P<0.0001)$, and ${ }^{* \star}$ NaCl-BCG vs. ALF-BCG $(P=0.0069)$. In all the experiments, for each ' $n$ ' value, ALFs from different donors were used.

supportive of accelerated immune responses in vaccinated mice. Moreover, mice vaccinated with $\mathrm{NaCl}$ - and ALF-exposed BCG visually had equal numbers of inflammatory foci and a similar increase in the number of lymphocytic cuffs in the lung at this time-point (Supplementary Figure S3A,B), indicating that the response to M.tb infection is accelerated in ALFexposed BCG-vaccinated mice.

At 250 DPI, the lungs of control (vehicle) mice had abundant cellular infiltration and inflammation, with $\sim 50 \%$ of the entire lung being involved (Figure 2a,c). Despite a significant extension in survival, the proportion of cellular infiltrate and lung inflammation in NaCl-exposed BCG-vaccinated mice was only moderately reduced relative to control mice (Figure 2a,c). Both control and $\mathrm{NaCl}$-exposed BCGvaccinated mice had macrophage-dominated cellular aggregates in the lung that consumed up to $40-50 \%$ of lung space (Figure 2a,c). In contrast, ALF-exposed BCG-vaccinated mice had significantly reduced lung cellular infiltrate relative to control and $\mathrm{NaCl}$-exposed BCG, with $\sim 75 \%$ of the lung space remaining uninvolved (Figure 2c). We also observed statistically significant decreases in the number of inflam- matory foci and lymphocytic cuffs per lung in animals vaccinated with ALF-exposed BCG (Supplementary Figure S3A,B). Furthermore, the smaller cell aggregates within the lung were dominated by lymphocytes (Figure 2a,c). Therefore, the enhanced control of M.tb at $250 \mathrm{DPI}$ and extended survival of ALF-exposed BCG was associated with a significant reduction in lung cellular accumulations, and those cells present in the lung were predominantly of the lymphocytic lineage.

We further validated our findings using the $\mathrm{C} 3 \mathrm{HeB} / \mathrm{FeJ}$ mouse strain (Supplementary Figure S3C,D and Supplementary Figure S4A-F), demonstrating that the capacity of ALFexposed BCG to confer better protection against M.tb infection, associated with reduce pulmonary cellular infiltration and inflammation, was common across mice of different genetic backgrounds and M.tb susceptibilities. Altogether our data indicate that not only is vaccination with ALF-exposed BCG superior to NaCl-exposed BCG at reducing the bacterial burden in targeted organs but it also can significantly reduce pulmonary inflammation and pathology that is typically associated with morbidity and mortality in $M$.tb-infected mice. 
a

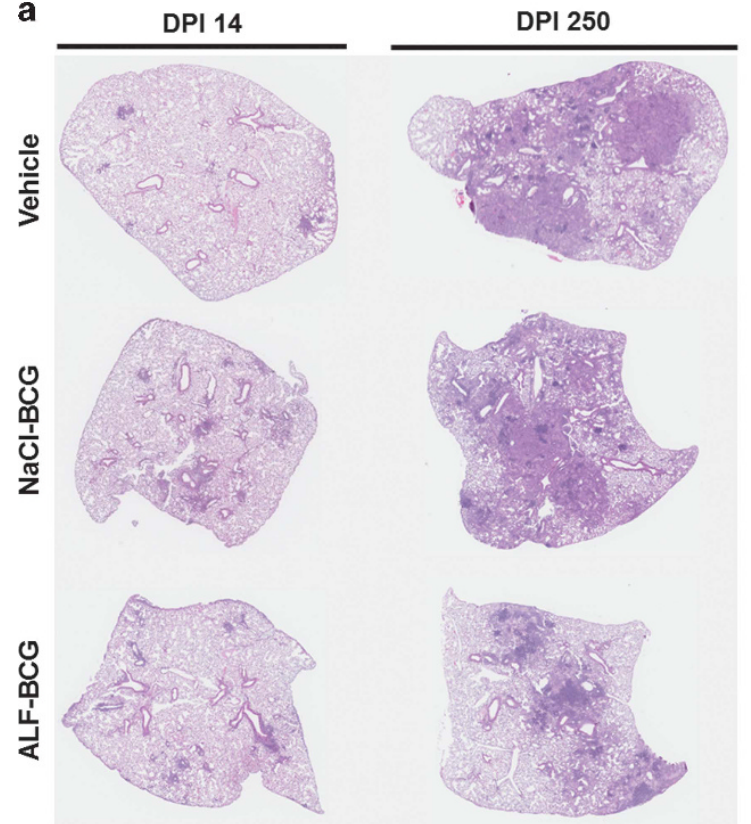

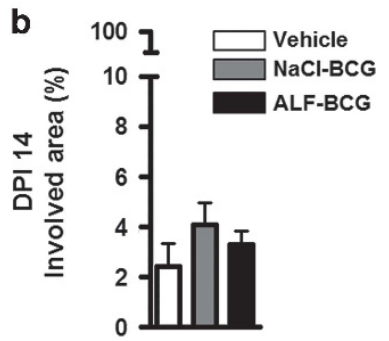

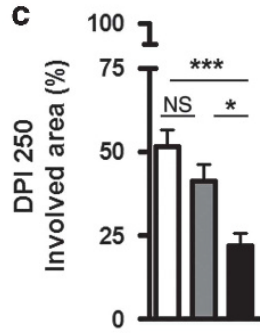

Figure 2 Vaccination with alveolar lining fluid (ALF)-exposed Bacillus Calmette-Guérin (BCG) reduces pulmonary inflammation in the Mycobacterium tuberculosis (M.tb) infected lung of vaccinated mice. C57BL/6J mice were vaccinated with NaCl-exposed BCG (NaCl-BCG; gray bars) or ALF-exposed BCG (ALF-BCG; black bars), or left unvaccinated (vehicle; open bars). Six weeks later, mice were infected with a low-dose aerosol of $M$.tb. (a) At 14 and 250 days post infection (DPI), C57BL/6J mice were killed and lungs fixed, embedded in paraffin, sectioned, and stained with hematoxylin and eosin to visualize tissue morphology. (b and $\mathbf{c}$ ) Pulmonary inflammation was quantified using Aperio Imagescope (Leica Biosystems, Buffalo Grove, IL). At least 12 distinct lobes were examined from $n=3$ with 4-5 mice per group per time-point in each experiment. A representative lobe is pictured; pooled data (involvement), mean \pm s.e.m.; one-way analysis of variance (ANOVA) with Tukey's post hoc test, ${ }^{*} P<0.05$ and ${ }^{* * *} P<0.001$; NS: not significant. In all the experiments, for each ' $n$ ' value, ALFs from different donors were used.

\section{Vaccination with ALF-exposed BCG enhances T-cell responses in the lung post M.tb challenge}

We first determined T-cell phenotype and function in the lung after BCG vaccination to elucidate how ALF-exposed BCG can modify the generation of T-cell effector and memory responses that would be in the lung during M.tb challenge. The total number (Figure 3) and the frequency (Supplementary Figure S5) of $\mathrm{CD}^{+}{ }^{+}$and $\mathrm{CD} 8{ }^{+} \mathrm{T}$ cells in the lungs of mice that received $\mathrm{NaCl}$-exposed or ALF-exposed BCG were equivalent, with no enhancement in the number or frequency of $\mathrm{CD} 4^{+}$or $\mathrm{CD} 8{ }^{+}$ effector and memory $\mathrm{T}$-cell subsets, or $\mathrm{T}$ cells capable of producing IFN $\gamma$. However, a significant increase in $\mathrm{CD} 8{ }^{+} \mathrm{T}$ cells expressing CD69 was observed in the lungs of mice vaccinated with ALF-exposed BCG.

We next characterized T-cell responses in the lungs of vehicle, $\mathrm{NaCl}$-, or ALF-exposed BCG vaccinated mice post $M$. $t b$ challenge. Mice were vaccinated, challenged with M.tb via aerosol, and killed at 14 DPI to characterize early T-cell responses in the lungs. In response to $M . t b$ challenge, the total number of $\mathrm{CD}^{+} \mathrm{T}$ cells increased significantly in the lung in the ALF-exposed BCG vaccinated mice relative to $\mathrm{NaCl}-$ exposed BCG (Figure 4a). Furthermore, we observed significant changes in the total number (Figure 4a-e) and frequency (Supplementary Figure S6) of CD8 ${ }^{+} \mathrm{T}$-cell subsets (for gating see Supplementary Figure S7). Mice vaccinated with ALF-exposed BCG had significantly increased numbers of $\mathrm{CD}^{+} \mathrm{T}$ cells that expressed a memory phenotype $\left(\mathrm{CCR} 7^{\text {neg }} \mathrm{CD} 62 \mathrm{~L}^{\mathrm{hi}} \mathrm{CD} 44^{\mathrm{hi}}\right.$ ) relative to vehicle and $\mathrm{NaCl}-$ exposed BCG (Figure 4b). Mice vaccinated with ALFexposed BCG also had an increased number of $\mathrm{CD} 8^{+} \mathrm{T}$ cells with the potential to secrete IFN $\gamma$, relative to both vehicle and $\mathrm{NaCl}$-exposed BCG (Figure 4d). We did not observe differences in the number of effector $\mathrm{T}$ cells $\left(\mathrm{CCR} 7{ }^{\text {neg }} \mathrm{CD} 62 \mathrm{~L}^{\mathrm{lo}} \mathrm{CD} 44^{\text {hi }}\right.$ ) in the lung (Figure 4c) and CD69 expression was also unchanged (Figure 4e).

The total number (Figure $\mathbf{4 a )}$ and frequencies (Supplementary Figure S6) of $\mathrm{CD}^{+} \mathrm{T}$ cells, $\mathrm{CD} 4^{+}$ effector, memory, and activated subsets, and $\mathrm{CD} 4^{+} \mathrm{T}$ cells capable of secreting IFN $\gamma$ was not significantly different between mice vaccinated with ALF-exposed BCG and vehicle control (Figure 4a-e and Supplementary Figure S6). A significant decrease in $\mathrm{CD}^{+}{ }^{+} \mathrm{T}$-cell frequency for IFN $\gamma$ was observed between ALF-exposed BCG and $\mathrm{NaCl}-$ exposed BCG, but this did not translate to a significant change in total cell number (Figure 4d). These data indicate that, in contrast to NaCl-exposed BCG, vaccination with ALF-exposed BCG has a significant impact on $\mathrm{CD} 8{ }^{+} \mathrm{T}$-cell numbers in the lung at day $14 \mathrm{DPI}$, and those cells expressed markers associated with a memory phenotype and had the capacity to secrete IFN $\gamma$. These phenotypes were much less apparent in the $\mathrm{CD} 4{ }^{+} \mathrm{T}$-cell subset.

$\mathrm{CD8}^{+} \mathrm{T}$ cells in the lungs of ALF-exposed BCG-vaccinated mice are required for enhanced protection against $M$.tb Having observed significant increases in CD ${ }^{+} \mathrm{T}$-cell numbers and activity in the lung of ALF-exposed BCG vaccinated mice, 

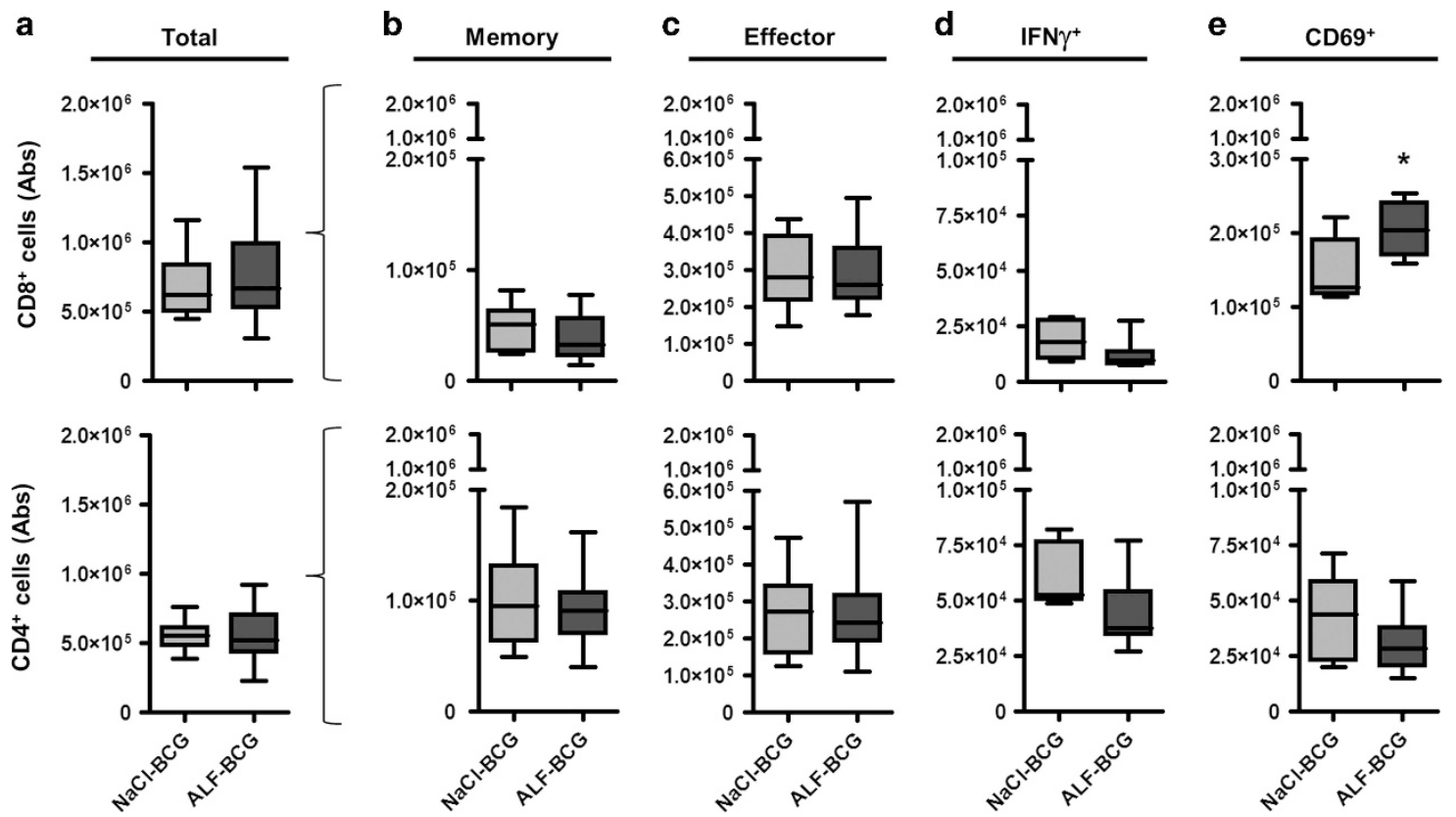

Figure 3 Vaccination with alveolar lining fluid (ALF)-exposed Bacillus Calmette-Guérin (BCG) preferentially stimulates CD8 ${ }^{+} \mathrm{T}$ cells in the lung. C57BL/6J mice were vaccinated with NaCl-exposed BCG (NaCl-BCG; white bars) or ALF-exposed BCG (ALF-BCG; black bars). Six weeks later, lungs were digested and single-cell suspension of cells were labeled with fluorescent antibodies specific for CD8 or CD4 in combination with CD69, CD62L, CCR7, CD44, and intracellular interferon- $\gamma$ (IFN $\gamma$ ). (a) Total number of CD8 ${ }^{+}$and CD4 ${ }^{+}$T cell in the lung. (b) Total number of CD8 ${ }^{+}$or CD4 ${ }^{+} \mathrm{T}_{\text {cells }}$ with a memory (CCR ${ }^{\text {neg }} \mathrm{CD} 62 \mathrm{~L}^{\text {hi }} \mathrm{CD} 44^{\text {hi }}$ ) phenotype. (c) Total number of CD8 ${ }^{+}$or CD4 ${ }^{+} \mathrm{T}$ cells with an effector $\left(\mathrm{CCR} 7^{\text {neg }} \mathrm{CD} 62 \mathrm{~L}^{\mathrm{lo}} \mathrm{CD} 44^{\mathrm{hi}}\right)$ phenotype. (d) Total number of $\mathrm{CD}^{+}{ }^{+}$or $\mathrm{CD} 4^{+}{ }^{\mathrm{T}}$ cells with the potential to produce IFN $\gamma$. (e) Total number of $\mathrm{CD} 8^{+}$or $\mathrm{CD} 4^{+} \mathrm{T}_{\text {cells expressing CD69. The absolute }}$ number of T cells in the lung is shown. Pooled data from $n=2$ with $4-5$ mice per group in each experiment, mean \pm s.e.m.; Student's $t$-test comparing $\mathrm{NaCl}-\mathrm{BCG}$ vs. ALF-BCG, ${ }^{\star} P<0.05$. Notice that $y$ axis scales among graphs are split different. In all the experiments, for each ' $n$ ' value, ALFs from different donors were used.

we explored whether the reduction in bacterial burden (Figure 1) was directly dependent on $\mathrm{CD}^{+} \mathrm{T}$ cells. Mice were vaccinated with vehicle, NaCl- or ALF-exposed BCG. Six weeks later, CD8 neutralizing antibodies or isotype controls were injected one day before M.tb infection and every 4 days thereafter. Mice were killed at 14 DPI to assess bacterial burden in the lung and spleen. Neutralizing antibodies successfully depleted $\mathrm{CD} 8{ }^{+} \mathrm{T}$ cells from the lung without affecting the total number of $\mathrm{CD} 4{ }^{+} \mathrm{T}$ cells (Figure 5a,b). $\mathrm{CD} 8{ }^{+} \mathrm{T}$-cell depletion had no observable effect on M.tb burden in the lung and spleen of mice vaccinated with the vehicle control (Figure 5c), confirming that $\mathrm{CD} 8{ }^{+} \mathrm{T}$ cells are not a major contributor at this stage of primary M.tb infection. ${ }^{18,19} \mathrm{CD} 8{ }^{+}$T-cell depletion also had no effect on $M . t b$ burden in the lung and spleen of mice that had received the NaCl-exposed BCG vaccination. ${ }^{20}$ However, in contrast to the $\mathrm{NaCl}$-exposed BCG group, $\mathrm{CD}^{+}$T-cell depletion of mice that had been vaccinated with ALF-exposed BCG lost the enhanced capacity to control $M . t b$ infection (Figure 5c), restoring M.tb burden to that of $\mathrm{NaCl}$-exposed BCG. These data demonstrate that activation and expansion of CD ${ }^{+}$T cells by ALF-exposed BCG is directly responsible for the enhanced protection induced by ALFexposed BCG.

We also analyzed the levels of IFN $\gamma$ and IL-12p40 in the lung of vaccinated and M.tb-infected mice with or without CD8 ${ }^{+}$ $\mathrm{T}$-cell depletion. Results showed significant increases in IFN $\gamma$ in the lungs of mice vaccinated with both $\mathrm{NaCl}$ - and ALF- exposed BCG (Figure 5d), but the highest level of IFN $\gamma$ were associated with ALF-exposed BCG vaccination. Similar to M.tb CFU, when ALF-BCG-vaccinated mice were depleted of CD8 ${ }^{+}$ T cells, IFN $\gamma$ production in response to $M$. $t b$ infection returned to levels comparable to $\mathrm{NaCl}$-exposed BCG. These data identify $\mathrm{CD}^{+} \mathrm{T}$ cells and IFN $\gamma$ as important contributors to the enhanced protection against $M$.tb challenge that is mediated by ALF-exposed BCG vaccination. We did not observe any differences in IL-12p40 between the vaccinated/CD8 ${ }^{+}$T-celldepleted groups (Figure 5d), further supporting the concept that $\mathrm{CD} 8{ }^{+} \mathrm{T}$ cells were a dominant source of IFN $\gamma .{ }^{21}$ Although we did not extend our neutralization studies to later stages of the disease, we can postulate that the reduction in pulmonary inflammation observed at later time-points of M.tb infection (Figure 2) is directly linked to early (and potentially extended) $\mathrm{CD}^{+}{ }^{+} \mathrm{T}$-cell activity in the lung.

\section{DISCUSSION}

Host immune responses to $M . t b$ and the requirements for its control have been well studied, ${ }^{22}$ yet immune mechanisms that result in efficient M.tb clearance from the host remain unknown. Here we show that vaccination with ALF-exposed BCG reduced early $M$.tb bacterial burden in the lung and spleen with superior control to that of conventional ( $\mathrm{NaCl}$-exposed) BCG. We purposefully chose an early time point as we predicted that ALF exposure of BCG would accelerate the control of M.tb, which is evident at day 14. ALF-exposed BCG 

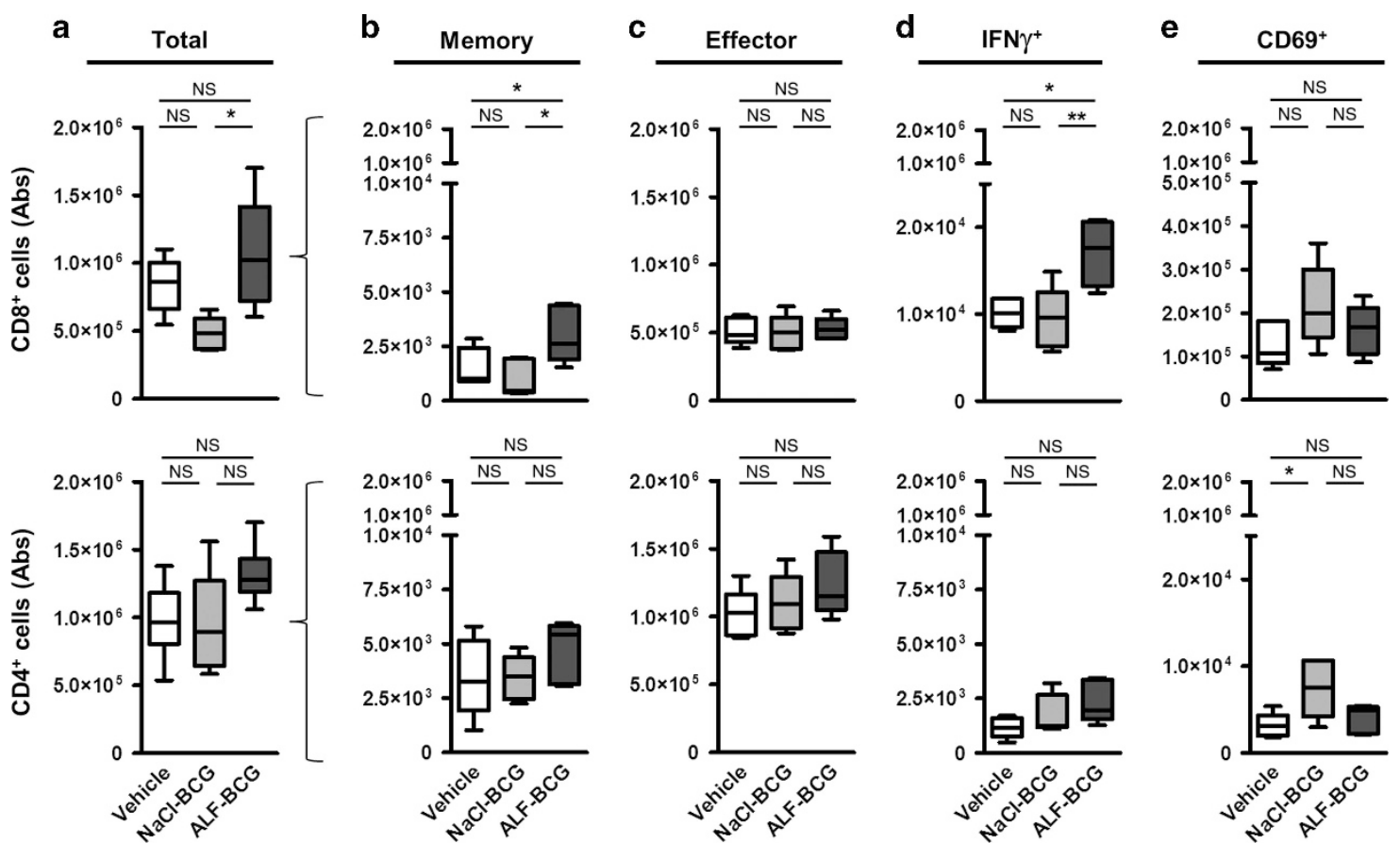

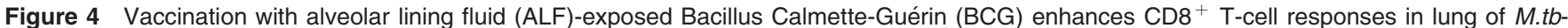
infected mice. C57BL/6J were vaccinated with vehicle (open bars), NaCl-exposed BCG (NaCl-BCG; gray bars), or ALF-exposed BCG (ALF-BCG; black bars). Six weeks post vaccination, mice were challenged with $M$.tb and killed at 14 days post infection (DPI) to characterize immune cell populations in the lung by flow cytometry. (a) Total number of $\mathrm{CD}^{+}$and $\mathrm{CD} 4^{+} \mathrm{T}$ cell in the lung. (b) Total number of $\mathrm{CD} 8^{+}$or $\mathrm{CD} 4^{+} \mathrm{T}$ cells with a memory $\left(\mathrm{CCR} 7^{\text {neg }} \mathrm{CD} 62 \mathrm{~L}^{\text {hi }} \mathrm{CD} 44^{\mathrm{hi}}\right)$ phenotype. (c) Total number of $\mathrm{CD} 8^{+}$or $\mathrm{CD} 4^{+} \mathrm{T}$ cells with an effector $\left(\mathrm{CCR} 7^{\text {neg }} \mathrm{CD} 62 \mathrm{~L}^{\text {lo }} \mathrm{CD} 44^{\mathrm{hi}}\right)$ phenotype. (d) Total number of $\mathrm{CD}^{+}{ }^{+}$or $\mathrm{CD} 4^{+}$T cells with the potential to produce interferon- $\gamma(\mathrm{IFN} \gamma)$. (e) Total number of CD8 ${ }^{+}$or CD4 ${ }^{+} \mathrm{T}^{-}$cells expressing CD69. Pooled experiment from $n=2$ with 4-5 mice per group, mean \pm s.e.m.; one-way analysis of variance (ANOVA) with Tukey's post hoc test, ${ }^{*} P<0.05$ and ${ }^{* *} P<0.01$; NS: not significant. Notice that $y$ axis scales among graphs are split different. In all the experiments, for each ' $n$ ' value, ALFs from different donors were used.

provided almost $1.0-\log _{10}$ protection at day 14 , whereas unexposed BCG only provided modest protection at this time point, supporting our prediction and hypothesis. Importantly, at later stages of M.tb infection, mice vaccinated with ALFexposed BCG retained the significantly lower bacterial burden in the lung and spleen compared with vehicle control, had reduced pulmonary inflammation in the lung, and extended host survival. These data support the concept that vaccine formulations capable of rapidly containing M.tb following infection are the most efficacious, ${ }^{23,24}$ where earlier control of $M . t b$ infection can translate into a reduction in pulmonary inflammation and M.tb bacterial burden at later stages and prolonged survival. We extend on this concept by implementing a vaccine strategy that is directed against the phenotype of $M . t b$ within the lung ALF, and demonstrate a clear enhancement of BCG-protective efficacy.

We extended our findings by establishing that $\mathrm{CD} 8^{+} \mathrm{T}$ cells make an important contribution to the degree of protection observed in ALF-exposed BCG-vaccinated mice throughout the first 14 days, with $\mathrm{CD} 8{ }^{+}$T-cell depletion reverting ALFexposed BCG protection (CFUs) back to that seen in mice vaccinated with $\mathrm{NaCl}$-exposed BCG. Indeed, ALF-exposed BCG vaccination leads to increased numbers of activated $\left(\mathrm{CD} 9^{+}\right) \mathrm{CD}^{+} \mathrm{T}$ cells within the lung, both $\mathrm{CD} 8{ }^{+} \mathrm{T}$ cells displaying a memory phenotype and $\mathrm{CD}^{+} \mathrm{T}$ cells with the potential to secrete IFN $\gamma$ in the lungs after M.tb challenge. This was further corroborated with our depletion $\mathrm{CD}^{+}$T-cell studies, which resulted in a reduction of IFN $\gamma$ levels in the lung. We anticipate that these activated $\mathrm{CD}{ }^{+} \mathrm{T}$ cells become resident memory $\mathrm{CD} 8{ }^{+} \mathrm{T}$ cells forming a reservoir that responds quickly to $M . t b .{ }^{25}$

The reduction of bacterial burdren in the lung and spleen of $M$.tb-infected mice receiving the ALF-exposed BCG vaccine was likely mediated by CD8 ${ }^{+}$T-cell-derived IFN $\gamma$, as IFN $\gamma$ concentrations in the lung was directly dependent on the presence or absence of $\mathrm{CD} 8^{+} \mathrm{T}$ cells despite similar numbers of $\mathrm{CD} 4{ }^{+} \mathrm{T}$ cells in the lung. IFN $\gamma$ is essential for the development of mycobacterial immunity, ${ }^{26,27}$ and $\mathrm{CD}^{+}$T-cell-derived IFN $\gamma$ has been previously shown to be important for protective immunity against $M . t b$ infection. ${ }^{21}$

We speculate that the reason why ALF-exposed BCG is better at priming $\mathrm{CD} 8{ }^{+} \mathrm{T}$ cells may be via mechanisms that promote macrophage bacterial killing and/or enhance antigen presentation. ${ }^{9}$ Our biochemical analyses reveal that, similar to $M . t b,{ }^{9}$ exposure to ALF partially removes ManLAM and TDM from the BCG cell wall surface. TDM is the most abundant and toxic glycolipid on the BCG cell wall and ManLAM is the most abundant lipoglycan. TDM impairs the development of adaptive immune responses by blocking the process of phagosome maturation, by decreasing the expression of 

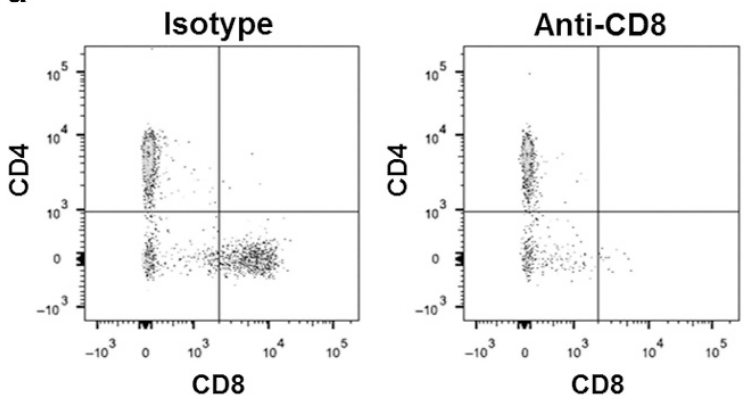

C

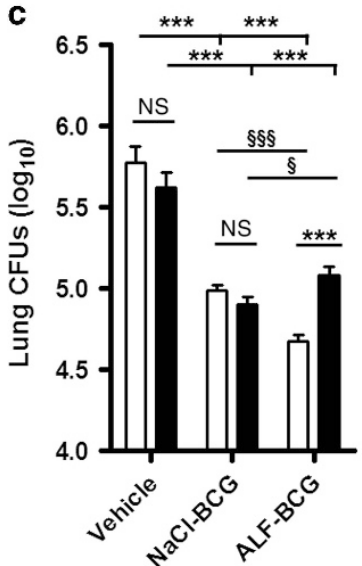

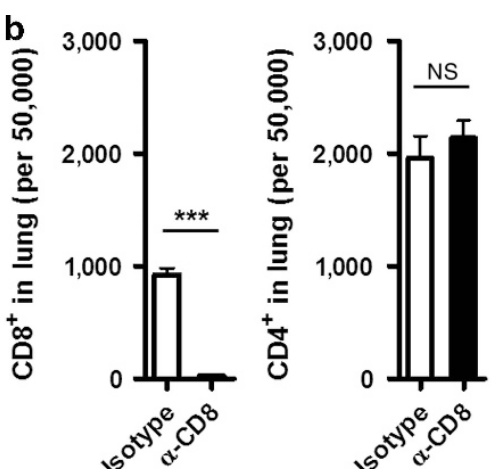

d

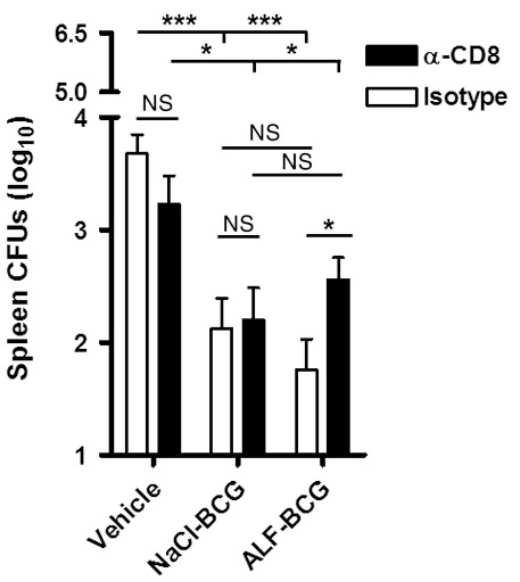

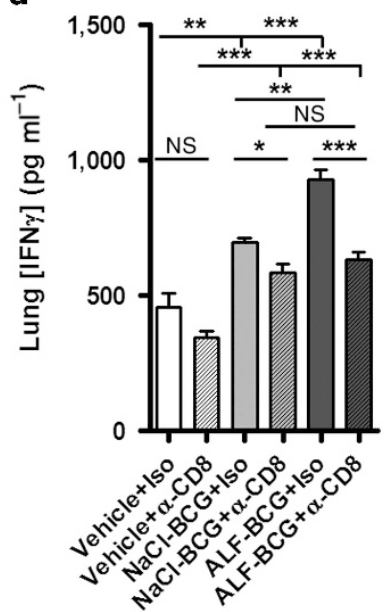

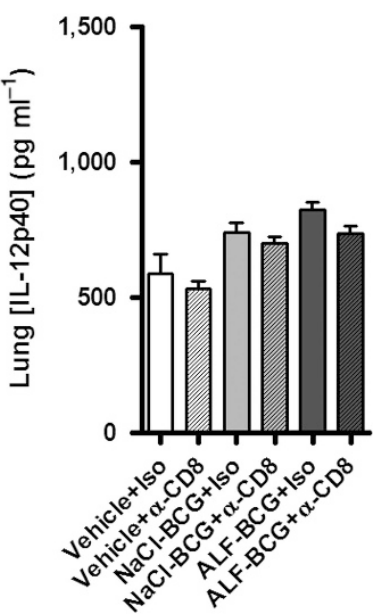

Figure 5 Reduction in bacterial burden associated with alveolar lining fluid (ALF)-exposed Bacillus Calmette-Guérin (BCG) is dependent on CD8 ${ }^{+}$ T-cell responses. C57BL/6J mice were vaccinated with vehicle, NaCl-exposed BCG, or ALF-exposed BCG. Six weeks after vaccination, mice were intraperitoneally injected with $500 \mu \mathrm{g}$ of anti-CD8a, or rat IgG2a and challenged with Mycobacterium tuberculosis (M.tb). Anti-CD8a or rat IgG2a was delivered 1 day before M.tb challenge and every 4 days thereafter. Mice were killed at 14 days post infection (DPI). (a) Schematic depicting effective depletion of $\mathrm{CD}^{+}{ }^{+} \mathrm{T}$ cells from the lung. (b) Quantification of $\mathrm{CD} 8^{+}$and $\mathrm{CD} 4^{+} \mathrm{T}$ cells in the lung of isotype-injected or anti-CD8a-injected mice. CD8 ${ }^{+} \mathrm{T}$ cells were depleted to $<20$ cells per 50,000 cells. Depletion of CD8 ${ }^{+}$T cells did not affect the number of CD4 ${ }^{+}$T cells in the lung. Pooled experiment from $n=2$ with 5 mice per group, mean \pm s.e.m.; student's $T$-test, ${ }^{* * *} P<0.001$. (c) M.tb CFUs were determined in the lung and spleen at 14 DPI. Pooled experiment from $n=2$ with 5 mice per group, mean \pm s.e.m.; one-way analysis of variance (ANOVA) with Tukey's post hoc test or Student's $t$-test (for single comparisons), ${ }^{\star} P<0.05$, ${ }^{\star \star} P<0.01,{ }^{\star \star \star} P<0.001,{ }^{\S} P<0.05$, and ${ }^{\S \$ \S} P<0.01$; NS: not significant. (d) Interferon- $\gamma$ (IFN $\gamma$ ) and IL-12p40 in the lung homogenates of vehicle-treated or -vaccinated mice with or without CD8 ${ }^{+}$T-cell depletion at $14 \mathrm{DPI}$. Representative experiment from $n=2$ with 5 mice per group, mean \pm s.e.m., one-way ANOVA with Tukey's post hoc test (for multiple groups) or Student's $t$-test (for single comparisons), ${ }^{\star} P<0.05$, ${ }^{\star \star} P<0.01$, and ${ }^{\star \star *} P<0.001$. In all the experiments, for each ' $n$ ' value, ALFs from different donors were used.

MHCII, CD40, CD80, and CD1d on macrophages, and by inhibiting macrophage-induced IL-12p40 (reviewed in ref. 28), a key cytokine required for IFN $\gamma$ secretion by T cells. Moreover, through the macrophage mannose receptor ManLAM limits phagosome maturation $^{29}$ and activates peroxisome proliferator-activated receptor- $\gamma$ modulating the macrophage inflammatory response..$^{30}$ ManLAM also downregulates the oxidative burst and inhibits $M . t b$-induced apoptosis (reviewed in ref. 28). Thus, ManLAM, as TDM, could alter T-cell proliferation and production of T-cell-derived IFN $\gamma$ by downregulating macrophage innate immune responses. Hence, surface removal of TDM and ManLAM from BCG by exposure to ALF could partially explain the improved adaptive immune response observed. Moreover, components of the complement system are also produced by alveolar epithelial cells ${ }^{31}$ and macrophages in the lung. ${ }^{32}$ It is reasonable to predict that the combination of ALF hydrolase-derived modifications and cell wall coating of
ALF-soluble components (complement, surfactant proteins, etc.) may have a role in the observed phenotype. Indeed, soluble components of serum such as complement can bind BCG, ${ }^{33}$ and thus potentially modify its interaction with the host. Whether complement deposition is the reason for increased protective efficacy of ALF-treated BCG is a current line of research in our lab.

The functionality of $\mathrm{CD} 8^{+} \mathrm{T}$ cells in BCG vaccination has not been thoroughly addressed. ${ }^{34} \mathrm{~A}$ recent study showed that direct inoculation of BCG into the mouse lung generated protective $\mathrm{CD} 8{ }^{+} \mathrm{T}$-cell-resident memory cells. ${ }^{35}$ Our observations are in agreement with this finding wherein we attained a similar phenotype by subcutaneous administration of ALFexposed BCG, which was simultaneously capable of reducing lung tissue damage. The mechanism(s) behind how $\mathrm{CD} 8^{+} \mathrm{T}$ cells contribute to the efficacy of ALF-exposed BCG could be through their cytotoxic function, ${ }^{36-38}$ their ability to stimulate 
other cells via secretion of specific immunomodulators, ${ }^{38}$ and/ or their ability to induce apoptosis of cells via the FasL-Fas pathways targeting cells expressing Fas. ${ }^{39}$ Any of these mechanisms could lead to the early M.tb control we observed, and we can also speculate that they contribute to the extended control of $M$.t $b$ infection and reduced pulmonary inflammation that are observed as $\mathrm{CD}^{+} \mathrm{T}$ cells have typically been associated with containment of $M . t b$ infection. ${ }^{40}$

It is well established that $\mathrm{CD} 4{ }^{+} \mathrm{T}$ cells mediate $0.5-1 \log _{10}$ reduction in $M$.tb bacterial burden in the lung and spleen of conventional BCG-vaccinated mice. ${ }^{41}$ Indeed, depletion of $\mathrm{CD}^{+}{ }^{+} \mathrm{T}$ cells did not restore M.tb CFU of NaCl- and ALFexposed BCG to that of vehicle control mice, suggesting that $\mathrm{CD}^{+}{ }^{+} \mathrm{T}$ cells mediated the $\mathrm{CFU}$ reduction observed between vehicle and NaCl-exposed BCG-vaccinated mice. We did not directly assess through depletion studies whether $\mathrm{CD} 4{ }^{+} \mathrm{T}$ cells contributed to the additional reduction in bacterial burden associated with ALF-exposed BCG vaccination because we did not observe any significant differences in $\mathrm{CD}^{+}$T-cell activation status. Likewise, we cannot completely rule out a role for protective antibodies and/or B-cell responses. ${ }^{42}$ It is possible that ALF treatment could reveal alternate and protective B-cell epitopes on BCG. Overall, our data support an important role for $\mathrm{CD} 8^{+} \mathrm{T}$ cells in the control of M.tb. In this context, $\mathrm{CD} 8{ }^{+} \mathrm{T}$ cells may be important mediators during the immediate immune response to M.tb in humans.

In addition to altering early immune events in the lung, ALFexposed BCG vaccination reduced disease manifestations. We observed minor increases in lung cell infiltration at earlier stages of infection in mice vaccinated with $\mathrm{NaCl}$ and ALFexposed BCG compared with vehicle control. Although changes did not reach significance, we attribute this to the development of immunological memory that allows for rapid responses to infection, ${ }^{43}$ and the expansion of an early and protective response to $M$.tb infection in vaccinated mice. Of more interest, however, was the significant decrease in lung inflammation in mice that were vaccinated with ALF-exposed BCG at later stages of infection (250 DPI). ALF-exposed BCG vaccination led to a greater reduction in tissue involvement and inflammation when compared with vehicle and conventional BCG-vaccinated animals. It is feasible that the early recruitment of IFN $\gamma^{+} \mathrm{CD}^{+}{ }^{+} \mathrm{T}$ cells induced by ALF-exposed BCG vaccination may have simply reduced $M . t b$ infection to low enough levels to induce less inflammation, but it is equally plausible that long-term control may have been a more active process. The relevance of reduced lung inflammation in mice vaccinated with ALF-exposed BCG relative to conventional BCG is currently unknown. We can associate it with the extended survival observed, although this is not an exact extrapolation because survival between mice vaccinated with ALF-exposed BCG was extended by only an additional 6 weeks compared with mice vaccinated with conventional $(\mathrm{NaCl}-$ exposed) BCG (the equivalent of about 3-5 years extended life in humans ${ }^{44}$ ). It is plausible that reduced pathology could also lead to altered morbidity, although these have not been directly studied, or simply reflect the limitations of working in the mouse model. Survival times varied within experimental groups by as much as 20 weeks; however, the distribution of survival observed is similar to what has been reported in the literature for C57BL/6J mice and is consistent across replicated experiments. ${ }^{45}$

We recognize that we introduced some variability into our studies by using different human ALF samples in each experiment. As an example is the protection from the $\mathrm{NaCl}$-exposed BCG group at 14 DPI in Figure 5c compared to that in Figure 1a. While we used the same human donor ALF for BCG and M.tb exposures (paired exposures in each ' $n$ ' value) before vaccination and infection (to ensure the same effects to the BCG and M.tb cell wall), each experiment was performed using a different human donor ALF to positively introduce human donor difference as an experimental variable (while working in an inbred mouse strain). We have previously published that ALF derived from different humans have slightly different effects on $M . t b^{9}$ resulting from variability in the amount of certain innate immune proteins (e.g., surfactant proteins, complement proteins, alveolar hydrolases) present within ALF among humans (ref. 10 and unpublished observations). Furthermore, as opposed to using frozen stocks of precalculated bacterial concentrations, we always generate single-cell suspensions of $M . t b$ from freshly agar plate grown bacteria to ensure that the mycobacterial cell wall remains intact. Indeed, growth in liquid medium has been shown to affect the presence/absence and abundance of certain cell wall molecules. ${ }^{46}$ Hence, it is important to recognize that even with the introduction of variability into our in vivo experimental system we were able to generate reproducible findings within and across experiments using BCG and M.tb exposed to different human ALFs, including our results in an independent mouse strain $(\mathrm{C} 3 \mathrm{HeB} /$ FeJ) that has a different susceptibility to M.tb infection.

In summary, human ALF contains an array of enzymes that modify the M.tb cell wall, ${ }^{9,14}$ which we hypothesize impact $M . t b$ uptake by phagocytic cells ${ }^{9,13,14,47}$ influencing the generation of adaptive immunity. In support of this, exposure of BCG to ALF increased the efficacy of BCG vaccination in terms of reducing bacterial burden and pulmonary inflammation, and the generation of $\mathrm{CD}^{+}{ }^{+} \mathrm{T}$ cells that are required for the increased efficacy of ALF-exposed BCG. Hence, our data demonstrate that human ALF exposure of BCG improves efficacy against M.tb challenge and suggest that manipulation of BCG, particularly with emphasis on its cell wall composition during infection within the lung, offers novel avenues to understand and improve on the development of protective immunity to mycobacteria. Furthermore, our data support that targeting the production of early and rapid CD8 ${ }^{+} \mathrm{T}$-cell responses in the lung may be important for containment of M.tb.

\section{METHODS}

Ethics statement. All experimental procedures with animals were approved by The Ohio State University Institutional Animal Care and Use Committee (IACUC protocol number: 2012A00000132-R1). For human subjects (to obtain bronchoalveolar lavage fluid), this study was 
carried out in strict accordance with US Code of Federal and Local Regulations (University Human Subjects Institutional Review Board (IRB) protocol number: 2008H0135), and Good Clinical Practice as approved by the National Institutes of Health/National Institute of Allergies and Infectious Diseases/Division of Microbiology and Infectious Diseases (NIH/NIAID/DMID protocol number: 12-0086). In this study only adult human subjects participated, and all of them provided written informed consent.

Mice. Specific pathogen-free, female mice aged 6-8 weeks of the $\mathrm{C} 57 \mathrm{BL} / 6 \mathrm{~J}$ or $\mathrm{C} 3 \mathrm{HeB} / \mathrm{FeJ}$ background were purchased from Jackson Laboratories (Bar Harbor, ME). Upon arrival, mice were supplied with sterilized water and chow ad libitum and acclimatized for at least 1 week before experimental manipulation. Mice were maintained in microisolator cages located in either a standard vivarium for all noninfectious studies or in a biosafety level three (ABSL-3) core facilities for all studies involving M.tb. Mice were divided into three groups: mock-vaccinated (vehicle), $\mathrm{NaCl}$-exposed BCG-vaccinated, or ALF-exposed BCG-vaccinated.

Growth conditions of M.tb and M. bovis BCG strain Pasteur. GFP-M.tb Erdman (provided by Dr Horwitz, UCLA, CA) and M. bovis BCG Pasteur strains (American Type Culture Collection (ATCC), Manassas, VA, no. 35734) were grown as described previously. ${ }^{9,13,14,47}$

Collection of human ALF and exposure to bacteria. Human ALF was obtained was obtained from human bronchoalveolar lavage fluid, concentrated to its physiological concentration within the human lung, and single-cell suspensions of $M$.t $b$ were exposed to human ALF as we reported previously. ${ }^{9,13,14}$ Bacteria were then washed with $0.9 \%$ $\mathrm{NaCl}$ to remove ALF and suspended at working concentrations for aerosol infections or subcutaneous injections with $M$.tb or M. bovis BCG, respectively.

Vaccination. Mice were subcutaneously injected in the scruff of the neck with $100 \mu \mathrm{l}$ of $0.9 \% \mathrm{NaCl}$ (saline, vehicle), $0.9 \% \mathrm{NaCl}$-exposed, or ALF-exposed M. bovis BCG Pasteur $\left(7.5 \times 10^{5} \mathrm{CFU}\right)$, diluted in sterile $0.9 \% \mathrm{NaCl}$. Mice were housed without further experimental manipulation for 6 weeks.

M.tb aerosol infection and CFU enumeration. Mice were infected aerogenically with a low dose of $M$.tb using the Glas-Col (Terre Haute, IN) inhalation exposure system as described previously. ${ }^{48}$ Bacterial burden was assessed at various DPI by culturing serial dilutions of organ homogenates onto Middlebrook 7H11 agar supplemented with OADC (oleic acid, albumin, dextrose, and catalase) as we have described previously. ${ }^{48}$ Colonies were enumerated following 14-21 days incubation at $37^{\circ} \mathrm{C}$. Data are expressed as the $\log _{10}$ value of the mean number of CFU recovered per organ ( $n=4-5$ mice). Lung homogenate were also plated onto OADC supplemented 7H11 agar containing $2 \mu \mathrm{g} \mathrm{ml}^{-1}$ of 2-thiophenecarboxylic acid hydrazide (Sigma-Aldrich, St. Louis, MO) to exclude and differentiate BCG growth from $M . t b .{ }^{49}$ Mice in survival studies were monitored over a period of 85 weeks. Mice were killed following a body condition scoring system ranging from 1 (emaciation) to 5 (obesity). Scores were determined by weekly visual and hands-on examination of each animal. Mice were killed when they reached a body condition scoring system of 2 or less. ${ }^{50}$

Lung cell isolation. Lung cells were isolated as described previously. ${ }^{51}$ For specific details, see Supplementary Materials.

Immunophenotyping by flow cytometry. Lung cells were prepared for flow cytometry as described previously. ${ }^{51}$ For specific details, see Supplementary Materials.

Enzyme-linked immunosorbent assay. Organ homogenates were analyzed for levels of IFN $\gamma$ and IL-12p40 by enzyme-linked immunosorbent assay following the manufacturer's instructions (BD Biosciences, San Jose, CA). ${ }^{52}$ Enzyme-linked immunosorbent assays were read on a Spectramax M2 Microplate reader (Molecular Devices LLC, Sunnyvale, CA). Surface ManLAM and TDM detection in whole bacteria after exposure to $\mathrm{NaCl}$ or human ALF is described in the Supplementary section.

Cell depletion. Cells depletions were performed as described previously. ${ }^{52}$ Briefly, $500 \mu \mathrm{g}$ anti-CD8 ${ }^{+}$(Clone: 53.6.72) depletion antibody (BioXCell, West Lebanon, $\mathrm{NH}$ ) or whole rat IgG2a (Clone: 2A3) (BioXCell) in $100 \mu \mathrm{l}$ were injected into the intraperitoneal cavity four times over a period of 2 weeks beginning one day before M.tb challenge. Lungs were digested to obtain whole lung cells and stained with fluorophore-conjugated antibodies for flow cytometry analysis to confirm $\mathrm{CD} 8{ }^{+}$T-cell depletion.

Histology. The middle right lung was isolated from each individual mouse and inflated with and stored in 10\% neutral-buffered formalin. Lung tissue was processed, sectioned, and stained with hematoxylin and eosin for light microscopy with lobe orientation designed to allow for maximum surface area of each lobe to be seen. Sections were examined in a blinded manner by a board-certified veterinary pathologist without prior knowledge of the experimental groups and evaluated according to severity, granuloma size and number, cellular composition, lymphocytic cuffing, and necrosis. Areas of cell aggregation were quantified using Aperio Imagescope at $\times 4$ magnification by calculating the total area of the involved tissue over the total area of the lobe for each individual mouse.

Total lipid extraction, thin layer chromatography, and densitometry analysis of TDM. For a detailed methodology see Supplementary Information.

Statistical analysis. Statistical significance was determined using the Prism 4 Software (GraphPad Software, San Diego, CA). The unpaired, two-tailed Student's $t$-test was used for two group comparisons. Multiple comparisons were analyzed using one-way analysis of variance with Tukey's post-test. Log-rank test was used to determine statistical significance of survival experiments. Statistical significance was reported as ${ }^{\star} P<0.05,{ }^{\star *} P<0.01$, or ${ }^{\star *}{ }^{\star} P<0.001$.

SUPPLEMENTARY MATERIAL is linked to the online version of the paper at http://www.nature.com/mi

\section{ACKNOWLEDGMENTS}

This work was supported by the National Institute of Allergy and Infectious Diseases at the National Institutes of Health (NIH/NIAID), grant numbers (AI073856 and AI093570) to J.B.T.; by the National Institute on Aging at the National Institute of Health (AG051428) supporting J.T. and J.B.T.; and by The Ohio State University (OSU) College of Medicine Systems in Integrative Biology Training Program-NIH/NIGMS T32-GM068412 and NIH/NIAID Al093570-S1 partially supporting J.I.M. This study was also partially supported by a CTSA Award No. UL1TR001070 from the National Center for Advancing Translational Sciences. The funders had no role in study design, data collection and interpretation, or the decision to submit the work for publication. The opinions expressed in this article are the author's own and do not reflect the view of the National Institutes of Health, the Department of Health and Human Services, or the United States government. We thank BEI Resources for providing anti-LAM CS35 Ab. We thank Dr Nagatoshi Fujiwara for providing anti-TDM Ab. We thank Drs Larry S. Schlesinger and Jian Zhang for their careful review of this manuscript. We also thank the University Laboratory Animal Resources and personnel and the Comparative Pathology and Mouse Phenotyping Shared Resource (P30 CA016058) in the Department of Veterinary Biosciences and the Comprehensive Cancer Center at OSU for their technical support. We would like to acknowledge the facilities and programmatic support of OSU Biosafety Level 3 Program.

\section{AUTHOR CONTRIBUTIONS}

J.I.M., A.P.H., C.H.C., V.D., J.T., and J.B.T. performed all experiments presented and all participated in getting data. J.I.M., M.D.W., and G.B. 
together with J.T. and J.B.T. participated in the planning of all the experiments presented and data interpretation. J.T. and J.B.T. also supervised the work and together with J.I.M. wrote this manuscript.

\section{DISCLOSURE}

The authors declared no conflict of interest.

c) 2018 Society for Mucosal Immunology

\section{REFERENCES}

1. World Health Organization. Global Tuberculosis Report, 2016. Available at:http://apps.who.int/iris/bitstream/10665/250441/1/9789241565394eng.pdf?ua $=1$.

2. Houben, R. M. \& Dodd, P. J. The global burden of latent tuberculosis infection: a re-estimation using mathematical modelling. PLoS Med. 13, e1002152 (2016).

3. Moliva, J. I., Turner, J. \& Torrelles, J. B. Prospects in Mycobacterium bovis Bacille Calmette et Guerin (BCG) vaccine diversity and delivery: Why does BCG fail to protect against tuberculosis?. Vaccine 33, 5035-5041 (2015).

4. Moliva, J. I., Turner, J. \& Torrelles, J. B. Immune responses to BCG vaccination: Why do they fail to protect against Mycobacterium tuberculosis?. Front. Immunol. 8, 407 (2017).

5. Abubakar, I. et al. Systematic review and meta-analysis of the current evidence on the duration of protection by bacillus Calmette-Guerin vaccination against tuberculosis. Health Technol. Assess. 17, 1-vi (2013).

6. Dockrell, H. M. Towards new TB vaccines: What are the challenges? Pathog. Dis. 74, ftw016 (2016).

7. Torrelles, J. B. \& Schlesinger, L. S. Integrating lung physiology, immunology, and tuberculosis. Trends Microbiol. 25, 688-697 (2017).

8. Whitsett, J. A., Wert, S. E. \& Weaver, T. E. Alveolar surfactant homeostasis and the pathogenesis of pulmonary disease. Annu. Rev. Med. 61, 105-119(2010).

9. Arcos, J., Sasindran, S. J., Fujiwara, N., Turner, J., Schlesinger, L. S. \& Torrelles, J. B. Human lung hydrolases delineate Mycobacterium tuberculosis-macrophage interactions and the capacity to control infection. J. Immunol. 187, 372-381 (2011).

10. Moliva, J. I., Rajaram, M. V., Sidiki, S., Sasindran, S. J., Guirado, E. \& Pan, $X$. J. et al. Molecular composition of the alveolar lining fluid in the aging lung. Age (Dordr) 36, 9633 (2014).

11. Wright, J. R. Immunoregulatory functions of surfactant proteins. Nat. Rev. Immunol. 5, 58-68 (2005).

12. Achkar, J. M. \& Casadevall, A. Antibody-mediated immunity against tuberculosis: implications for vaccine development. Cell Host Microbe 13, 250-262 (2013).

13. Arcos, J. et al. Lung mucosa lining fluid modifies Mycobacterium tuberculosis to reprogram human neutrophil killing mechanisms. J. Infect. Dis. 212, 948-958 (2015).

14. Arcos, J. et al. Mycobacterium tuberculosis cell wall released fragments by the action of the human lung mucosa modulate macrophages to control infection in an IL-10-dependent manner. Mucosal Immunol. 10, 12481258 (2017).

15. Horvath, C. N., Shaler, C. R., Jeyanathan, M., Zganiacz, A. \& Xing, Z. Mechanisms of delayed anti-tuberculosis protection in the lung of parenteral BCG-vaccinated hosts: a critical role of airway luminal T cells. Mucosal Immunol. 5, 420-431 (2012).

16. Cyktor, J. C., Carruthers, B., Stromberg, P., Flano, E., Pircher, H. \& Turner, J. Killer cell lectin-like receptor G1 deficiency significantly enhances survival after Mycobacterium tuberculosis infection. Infect. Immun. 81, 1090-1099 (2013).

17. Ozeki, Y., Hirayama, Y., Takii, T., Yamamoto, S., Kobayashi, K. \& Matsumoto, S. Loss of anti-mycobacterial efficacy in mice over time following vaccination with Mycobacterium bovis Bacillus Calmette-Guerin. Vaccine 29, 6881-6887 (2011).

18. Turner, J. etal. CD8- and CD95/95L-dependent mechanisms of resistance in mice with chronic pulmonary tuberculosis. Am. J. Respir. Cell. Mol. Biol. 24, 203-209 (2001).

19. Xing, Z., Wang, J., Croitoru, K. \& Wakeham, J. Protection by CD4 or CD8 T cells against pulmonary Mycobacterium bovis Bacillus Calmette-Guerin infection. Infect. Immun. 66, 5537-5542 (1998).

20. Chen, C. Y. et al. A critical role for CD8 Tcells in a nonhuman primate model of tuberculosis. PLoS Pathog. 5, e1000392 (2009).
21. Tascon, R. E., Stavropoulos, E., Lukacs, K. V. \& Colston, M. J. Protection against Mycobacterium tuberculosis infection by $\mathrm{CD} 8+T$ cells requires the production of gamma interferon. Infect. Immun. 66, 830-834 (1998).

22. Flynn, J. L. \& Chan, J. Immunology of tuberculosis. Annu. Rev. Immunol. 19, 93-129 (2001).

23. Ordway, D. et al. Influence of Mycobacterium bovis BCG vaccination on cellular immune response of guinea pigs challenged with Mycobacterium tuberculosis. Clin. Vaccine Immunol. 15, 1248-1258 (2008).

24. Khader, S. A. et al. IL-23 and IL-17 in the establishment of protective pulmonary $\mathrm{CD} 4+\mathrm{T}$ cell responses after vaccination and during Mycobacterium tuberculosis challenge. Nat. Immunol. 8, 369-377 (2007).

25. Connor, L. M. et al. A key role for lung-resident memory lymphocytes in protective immune responses after BCG vaccination. Eur. J. Immunol. 40, 2482-2492 (2010).

26. Flynn, J. L., Chan, J., Triebold, K. J., Dalton, D. K., Stewart, T. A. \& Bloom, B. R. An essential role for interferon gamma in resistance to Mycobacterium tuberculosis infection. J. Exp. Med. 178, 2249-2254 (1993).

27. Cooper, A. M., Dalton, D. K., Stewart, T. A., Griffin, J. P., Russell, D.G. \& Orme, I. M. Disseminated tuberculosis in interferon gamma genedisrupted mice. J. Exp. Med. 178, 2243-2247 (1993).

28. Torrelles, J. B. Broadening our view about the role of Mycobacterium tuberculosis cell envelope components during infection: a battle for survival. In Understanding Tuberculosis-Analyzing the Origin of Mycobacterium tuberculosis Pathogenicity, Vol 12 (Cardona, P. J., ed) 77-122 (Intech, Rijeka, Croatia, 2012).

29. Kang, P. B. et al. The human macrophage mannose receptor directs Mycobacterium tuberculosis lipoarabinomannan-mediated phagosome biogenesis. J. Exp. Med. 202, 987-999 (2005).

30. Rajaram, M. V., Brooks, M. N., Morris, J. D., Torrelles, J. B., Azad, A. K. \& Schlesinger, L. S. Mycobacterium tuberculosis activates human macrophage peroxisome proliferator-activated receptor gamma linking mannose receptor recognition to regulation of immune responses. J. Immunol. 185, 929-942 (2010).

31. Strunk, R. C., Eidlen, D. M. \& Mason, R. J. Pulmonary alveolar type II epithelial cells synthesize and secrete proteins of the classical and alternative complement pathways. J. Clin. Invest. 81, 1419-1426 (1988).

32. Cole, F. S., Matthews, W. J. Jr., Rossing, T. H., Gash, D. J., Lichtenberg, N. A. \& Pennington, J. E. Complement biosynthesis by human bronchoalveolar macrophages. Clin. Immunol. Immunopathol. 27, 153159 (1983).

33. Carroll, M. V., Lack, N., Sim, E., Krarup, A. \& Sim, R. B. Multiple routes of complement activation by Mycobacterium bovis BCG. Mol. Immunol. 46, 3367-3378 (2009).

34. Abebe, F. Is interferon-gamma the right marker for bacille Calmette-Guerininduced immune protection? The missing link in our understanding of tuberculosis immunology. Clin. Exp. Immunol. 169, 213-219 (2012).

35. Perdomo, C. et al. Mucosal BCG vaccination induces protective lungresident memory $T$ cell populations against tuberculosis. $m B i o ~ 7$, pii: e01686-16 (2016).

36. Turner, J. \& Dockrell, H. M. Stimulation of human peripheral blood mononuclear cells with live Mycobacterium bovis BCG activates cytolytic CD8 + T cells in vitro. Immunology 87, 339-342 (1996).

37. Murray, R. A. et al. Bacillus Calmette Guerin vaccination of human newborns induces a specific, functional $\mathrm{CD} 8+\mathrm{T}$ cell response. J. Immunol. 177, 5647-5651 (2006).

38. Bevan, M. J. Helping the CD8(+) T-cell response. Nat. Rev. Immunol. 4, 595-602 (2004).

39. Oddo, M., Renno, T., Attinger, A., Bakker, T., MacDonald, H. R. \& Meylan, P. R. Fas ligand-induced apoptosis of infected human macrophages reduces the viability of intracellular Mycobacterium tuberculosis. J. Immunol. 160, 54485454 (1998).

40. van Pinxteren, L. A., Cassidy, J. P., Smedegaard, B. H., Agger, E. M. \& Andersen, P. Control of latent Mycobacterium tuberculosis infection is dependent on CD8 T cells. Eur. J. Immunol. 30, 3689-3698 (2000).

41. Andersen, P. Effective vaccination of mice against Mycobacterium tuberculosis infection with a soluble mixture of secreted mycobacterial proteins. Infect. Immun. 62, 2536-2544 (1994).

42. Prados-Rosales, R. etal. Enhanced control of Mycobacterium tuberculosis extrapulmonary dissemination in mice by an arabinomannan-protein conjugate vaccine. PLoS Pathog. 13, e1006250 (2017). 


\section{ARTICLES}

43. Shen, Y. et al. Adaptive immune response of Vgamma2Vdelta2 + T cells during mycobacterial infections. Science 295, 2255-2258 (2002).

44. Dutta, S. \& Sengupta, P. Men and mice: relating their ages. Life Sci. 152, 244-248 (2016).

45. Sun, R. et al. Novel recombinant BCG expressing perfringolysin $O$ and the over-expression of key immunodominant antigens; pre-clinical characterization, safety and protection against challenge with Mycobacterium tuberculosis. Vaccine 27, 4412-4423 (2009).

46. Prados-Rosales, R. et al. The type of growth medium affects the presence of a mycobacterial capsule and is associated with differences in protective efficacy of BCG vaccination against Mycobacterium tuberculosis. J. Infect. Dis. 214, 426-437 (2016).

47. Scordo, J. et al. Mycobacterium tuberculosis cell wall fragments released upon bacterial contact with the human lung mucosa alter the neutrophil response to infection. Front. Immunol. 8, 307 (2017).
48. Cyktor, J. C., Carruthers, B., Kominsky, R. A., Beamer, G. L., Stromberg, P. \& Turner, J. IL-10 inhibits mature fibrotic granuloma formation during Mycobacterium tuberculosis infection. J. Immunol. 190, 2778-2790 (2013).

49. Flaherty, D. K., Vesosky, B., Beamer, G. L., Stromberg, P. \& Turner, J. Exposure to Mycobacterium avium can modulate established immunity against Mycobacterium tuberculosis infection generated by Mycobacterium bovis BCG vaccination. J. Leukoc. Biol. 80, 1262-1271 (2006).

50. Ullman-Cullere, M. H. \& Foltz, C. J. Body condition scoring: a rapid and accurate method for assessing health status in mice. Lab. Anim. Sci. 49, 319-323 (1999).

51. Canan, C. H. et al. Characterization of lung inflammation and its impact on macrophage function in aging. J. Leukoc. Biol. 96, 473-480 (2014).

52. Cyktor, J. C., Carruthers, B., Beamer, G. L. \& Turner, J. Clonal expansions of CD8(+) T cells with IL-10 secreting capacity occur during chronic Mycobacterium tuberculosis infection. PLOS ONE 8, e58612 (2013). 